\title{
Blade Tip Clearance Measurement USing MICROWAVE SENSING SYSTEM
}

\author{
Anwesha Dutta ${ }^{1}$, Shivangi ${ }^{1}$, J. Valarmathi $^{1}$ \\ ${ }^{1}$ School of Electronics Engineering, VIT University, Vellore, TamilNadu, India
}

\begin{abstract}
Tip clearance is the gap between the tip of a rotating airfoil blade and stationary engine casing in gas turbine. The blades are exposed to centrifugal force because of high rotational speed, that's why clearance variations occur during a flight. Tip clearance is inversely proportional to fuel efficiency (i.e.), large tip clearance value will reduce the efficiency. Hence, tip clearance value should be small but still shouldn't even brush the casing. Many sensing strategies like capacitance, optics, laser, eddy current and temperature are used to measure tip clearance. Many Organizations are using capacitive sensing system for tip clearance measurement, in which the effect of noise is more at high temperature. In this paper, Microwave measurement system based on short-range radar principle has been proposed. Sensor outputs are synthesized and analysed for different noise levels. It is advantageous over the other technologies because it can withstand extremely high temperature.
\end{abstract}

\section{KEYWORDS}

Gas Turbine, Blade tip clearance (BTC),Microwavesensing,waveguide resonator, In-phase and quadrature component.

\section{INTRODUCTION}

A gas turbine is a modification of combustion engine. The combustion engine or the gas turbine works under tremendous amount of pressure and temperature. The rotating blades in the turbines are subjected to centrifugal force, which acts outwards in the radial direction. This causes the blades to vibrate. The high operating temperature and pressure aggravates the vibrations, which often cause significant blade tip clearance changes.

Blade tip clearance is the distance between the stationary engine casing and the rotating airfoil blade. Due to variation in thermal and mechanical loads on the rotating blade and stationary structures, blade tip clearance keeps varying. This problem plagues both aero-engines and landbased turbo-engines. However, tip clearance monitoring is more important for aero-engines due to rapid change in the frequency of operation and varying nature of aerodynamics and inertial loads during flight. The efficiency of the gas turbine engine is inversely related to the tip clearance between the engine casing and the rotating blade. According to the literature survey it was found out that with just $0.0254 \mathrm{~mm}$ reduction in tip clearance, the fuel efficiency increases by almost $1 \%$. Hot high-pressure gases escape through the blade tip clearance. If tip clearance is small, the blades might rub the casing and cause catastrophic disintegration of the turbine. On the other hand, large tip clearance will allow more amount of gas to pass through which will lead to high leakage of gas resulting in reduction in fuel efficiency. Thus it is required to strike a balance between the two and maintain an optimum blade tip clearance. This necessitates the continuous monitoring of blade tip clearance. 
Various sensing strategies have been proposed over the years to measure blade tip clearance. Sensing systems based on optics, capacitance, eddy current and microwave are present for tip clearance measurement. Some industries are currently using capacitive sensing system for blade tip clearance measurement. This technique is greatly affected by noise. Hence, an alternative solution for clearance measurement is necessary. The objective of the project is to determine a suitable measuring system to be used for tip clearance measurement in Gas turbine engines and analyse its performance by simulation.

Different types of sensors are used to measure the tip clearance values in gas turbine engine. The sensing strategies used for the purpose of measuring are based on microwaves sensing system, capacitance probe, laser, optics and eddy currents.

LASER based optic probe can measure the blade tip clearance. A LASER beam is projected on the rotating blades. The blades reflect the beam and the blade tip clearance is measured by the position shift of the reflected LASER beam compared to the transmitted beam.

A capacitive sensor involves placing a probe in the engine casing. The tip of the blade and an electrode mounted on the casing thus forms a parallel capacitor. As the tip clearance varies, the capacitance value changes and the tip clearance can be measured [5].

In Eddy current tip clearance sensors, a magnetic circuit is mounted in the casing. The magnetic field reaches the rotating blade. Eddy-currents is induced in the rotating blades that create a magnetic field which affects the magnetic circuit mounted on the casing and the rotating blade tip clearance can be detected and monitored [6].

These sensing strategies have many shortcomings which are listed below: Optical sensors are expensive, sensitive to the operating environment and expensive. Capacitive sensors suffer from bandwidth limitation and low resolution. In Eddy current based sensors, permanent magnets are used for input. Electromagnetic techniques could change the properties of the magnet [4].

Sensor probes should be capable of tolerating vibration, large temperature variations, and presence of dirt and corrosive gases throughout its operating lifetime. This severe nature of the environment restricts the extended use of most technologies. Microwave sensors has many advantageous over the other sensors as listed below:

Microwave sensors have the ability to operate at extremely high temperatures. They are unaffected by presence of contaminants. The microwave radiations have high frequency and that is why provides excellent propagation even in such hostile environment [1].

The sensing strategy is based on measurement of the phase change between the transmitted and the received electromagnetic waves. Microwave sensors simplify this since metals reflect microwave satisfactorily.

However it has some disadvantages too. Microwave sensors are expensive to manufacture. These sensors operate at high operating frequency due to which complicated calibration and advanced signal processing is needed which increases the system complexity.

It has been found that microwave sensing is superior to the existing sensing techniques owing to its ability to withstand high temperature and pressure. We have proposed a blade tip clearance measurement based on microwave waveguide sensors. Its operation has been simulated using MATLAB.

This paper is organized as follows; Section II gives a brief introduction about the operation of a 
Gas turbine engine. The microwave sensing technique has been dealt with in Section III and an algorithm for the same has been proposed. The simulation results are presented in Section IV. Finally, conclusions are given in Section V.

\section{WorkingPrincipleOf A Gas Turbine Engine}

In a steam turbine engine, high-pressure steam is made to rotate the turbine and energy is extracted from it. In the same way, in a gas turbine engine the fuel is burned in the combustion unit and the high-pressure gas thus formed is used to drive the turbine. The gas turbine engine has four major sections-The inlet, compressor, combustion and the turbine section as shown in figure 1. Compressor section compresses the incoming air to high pressure. The fuel is burned to produce high-pressure, high velocity gas in the combustionchamber. The energy from the highpressure, high-velocity gas flowing out from the combustion chamber is extracted in turbine region.

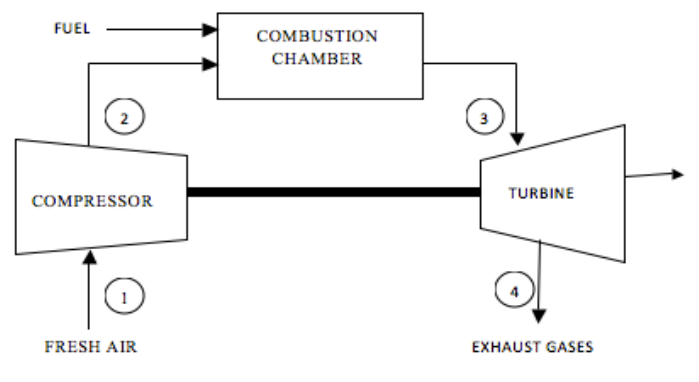

Figure1.Functionalblock diagram of gas turbine engine.

Gas turbine employs a thermodynamic procedure called as the "Brayton cycle". In this cycle, the efficiency improves as the pressure difference across the system increases. The compressor section compresses the working fluid that is, air. This compression is of adiabatic type since no heat gain or loss occurs. This compressed air is then mixed with fuel and burned using the fuel burners in the combustion unit. The hot, high-speed gas thus formed, gets expanded through the turbine to generate power. This power produced in the turbine is used to run the compressor and the rest is used to run the supporting equipment [7].

As shown in figure 1, the turbine and the compressor are attached to the same shaft. As the gas turbine speeds up, the compressor also speeds up forcing more air to flow through the combustion chamber. This increases the rate of burning of the fuel, which increases the amount of gas flowing through gas turbine increasing its speed even more. Increasedrotational speed in turbine results in higher centrifugal force and more clearance variations. This necessitates the control of this uncontrolled positive feedback. When blade tip clearance reaches an alarming value, the turbine speed has to be reduced. Limiting the amount of fuel fed to the turbine can do this.

\section{Microwave Sensing System Overview}

The microwave sensors transmit a continuous frequency microwave signal towards the rotating turbine blades and receive the reflected signal. At resonance, when the angular velocity of the blades becomes equal to the frequency of the transmitted signal, the electromagnetic wave is able to detect the blade tip and interacts with the tip of the blade, while out of resonance; it is unable to detect the blade, producing just the reference signal. This can be processed in a phase-based signal-processing module to estimate the tip clearance [2]. 
The microwave sensing system is based on short-range radar principle. The microwave generator sends a continuous wave microwave signal towards the blade. The measurement system uses microwave probes, which can tolerate high temperature. The probes are mounted on the engine casing of the turbine, so that it is able to see the blade tip easily. The sensing probe consists of a resonant open-ended cylindrical waveguide protected by the ceramic cap. It acts an antenna, which is capable of transmitting the continuous wave generated by microwave signal generator as well as receiving back the reflected wave by the tip of the blade. The generated continuous wave is transmitted to the probe passing through the circulator again gets reflected back by the blade tip. Two radio frequency mixers are used to calculate the In-phase and Quadrature component of the reflected wave. Two mixers compare the received signal reflected back from blade tip and the reference signal sent by the microwave signal generator. By processing both components phase is determined. The phase difference between the transmitted and received signal is directly proportional to the distance between the tip of the blade and engine casing where probe is mounted. The relation between the calculated phase $\theta$ and the tip clearance value $\beta$ is given by Equation (1) which shows that measured phase depends inversely on the frequency of the operation which proves that microwave system can effectively measure small tip clearance.

$$
\beta=\frac{\theta \times c}{4 \pi f}
$$

Where $\beta$ is the tip clearance and $\theta$ is the calculated phase shift between transmitted and reflected wave and $\mathrm{c}$ is the speed of light.

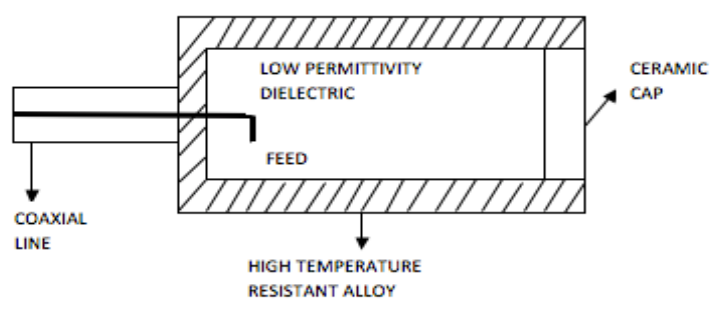

Figure 2.Microwave waveguide resonator probe structure.

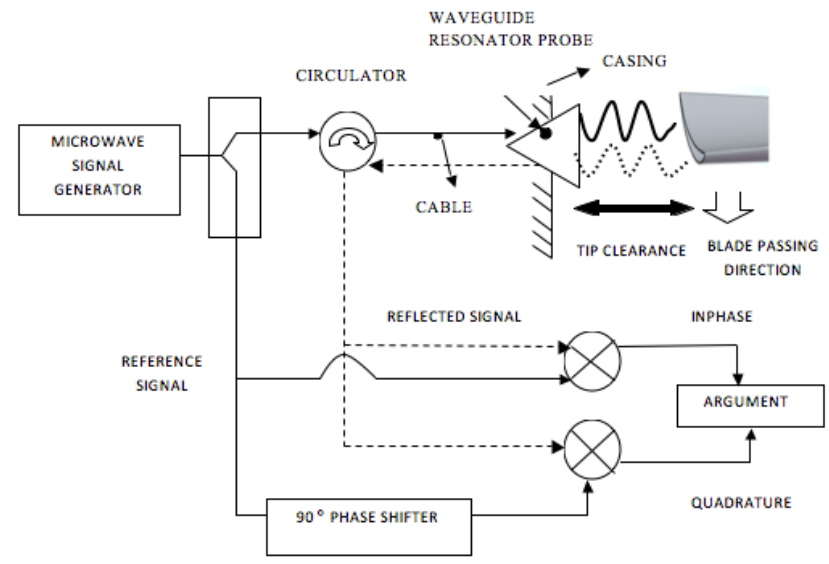

Figure 3. Block diagram of microwave sensing system.

To find the phase shift between the transmitted and received wave first we have to find the Inphase and Quadrature components related to the reference signal. In-phase component is the 
component that is in the same axis as the reference signal. Quadrature component is perpendicular to the reference axis. Letcos $\left(\omega_{c} t\right)$ be the transmitted wave. After reflection from the rotating blade the transmitted wave will undergo some attenuation and phase change.Thus the received signal becomes $a(t) \cos \left(\omega_{c} t+\theta(t)\right)$ where, $\theta(t)$ is the phase shift occurring between the transmitted and reflected wave and $a(t)$ is the time-varying attenuation factor.

To determine the Quadrature component the received wave has to be multiplied with the transmitted wave (taken as reference)

In-phase component is given by

$$
\begin{aligned}
& I=P_{\text {rec }}(t) \cos \left(\omega_{c} t\right)=a(t) \cos \left(\omega_{c} t+\theta(t)\right) \cos \left(\omega_{c} t\right) \\
& =a(t) \frac{1}{2}\left[\left(\cos \left(\theta(t)+2 \omega_{c} t\right)\right)+\cos \theta(t)\right]
\end{aligned}
$$

Filter out $a(t) \frac{1}{2}\left[\left(\cos \left(\theta(t)+2 \omega_{c} t\right)\right)\right]$ component from equation (2), using a low pass filter, we get

$$
I_{\text {filtered }}=a(t) \frac{1}{2}[\cos \theta(t)]
$$

Quadrature component is obtained by multiplying the received signal by the $90^{\circ}$ shifted reference signal.

Quadrature component is given by

$$
\begin{aligned}
& \qquad=P_{r e c}(t) \sin \left(\omega_{c} t\right)=a(t) \cos \left(\omega_{c} t+\theta(t)\right) \sin \left(\omega_{c} t\right) \\
& =a(t) \frac{1}{2}\left[\left(\cos \left(\theta(t)+2 \omega_{c} t\right)\right)-\sin \theta(t)\right]
\end{aligned}
$$

Filter out $a(t) \frac{1}{2}\left[\left(\cos \left(\theta(t)+2 \omega_{c} t\right)\right)\right]$ component from equation (4), using a low pass filter, we get

$Q_{\text {filtered }}=a(t) \frac{1}{2}[\sin \theta(t)]$

Phase shift can be determined by

$$
\begin{aligned}
& \text { Phase shift }=\tan ^{-1}\left[\frac{\text { Quadrature component }}{\text { Inphase component }}\right]=\tan ^{-1}\left[\frac{Q_{\text {filtered }}}{I_{\text {filtered }}}\right] \\
& =\tan ^{-1}\left[\frac{\sin \theta(t)}{\cos \theta(t)}\right] \\
& =\tan ^{-1}[\tan \theta(t)] \\
& =\theta(t) \quad
\end{aligned}
$$

Substitute equation (6) in equation (1) to find $\beta$, the tip clearance, 


\section{Simulation Results}

The simulation tool used for the purpose of this paper is MATLAB. The parameters taken for simulation are as follows. Frequency of operation is taken to be $24 \mathrm{GHz}$. Introduced phase shift is $-p i / 2$ to $+p i / 2$.Received signal magnitude is assumed to be varying from 0.1 to 1 .

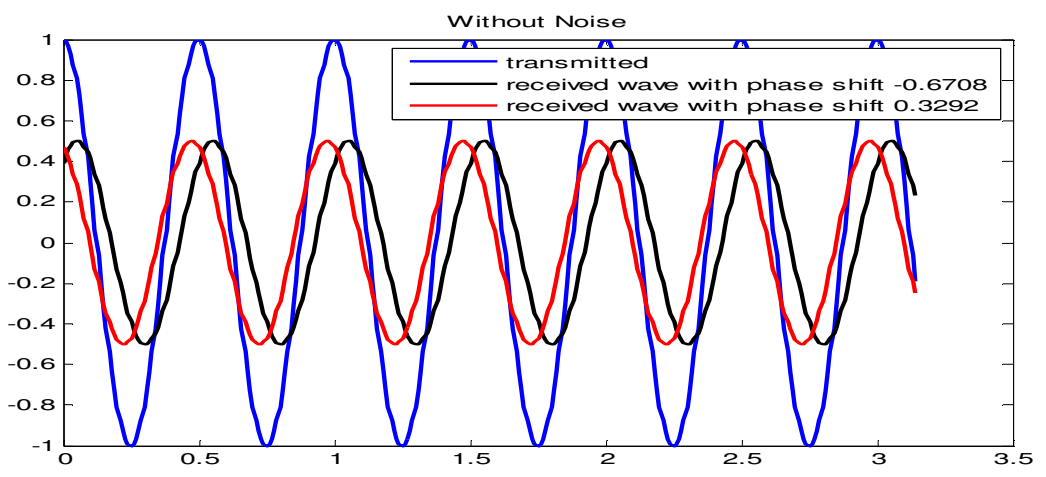

Figure 4.Plot of Transmitted wave, reflected wave without noise.

The transmitted wave simulation and the reflected wave simulation for two different phase shifts have been plotted for illustration purpose. As we can observe, in this simulation we have considered the reflected wave to be attenuated by half when compared to the transmitted.

In practical case, noise corrupts the reflected wave. The simulation for transmitted and noise affected reflected wave for different phase shifts is shown below.

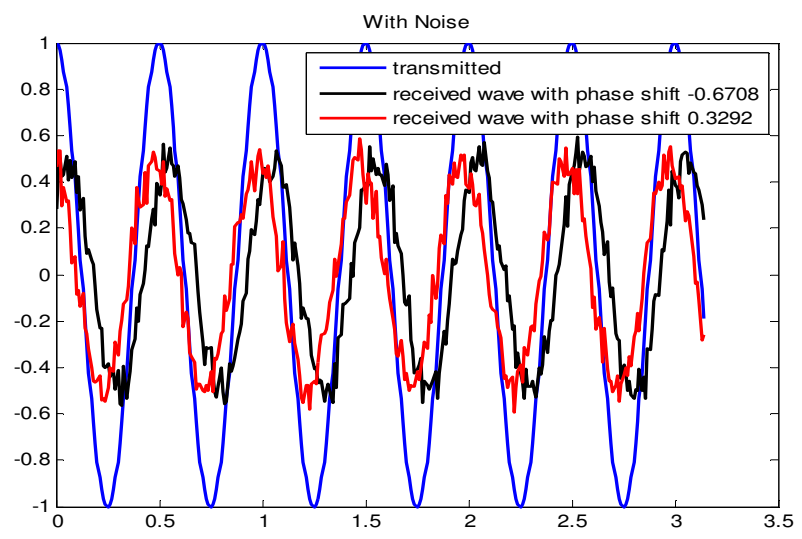

Figure 5 Plot of Transmitted wave, reflected wave with noise.

The estimated tip clearance is plotted with respect to number of phase shift. As expected, the tip clearance varies linearly with the phase shift between transmitted and reflected wave.

Estimated phase shift is same as the introduced phase shift when no noise is corrupting the received wave. In the presence of noise, estimated phase deviates a little from the ideal case. Similarly, estimated tip clearance with and without noise is almost equal. The tip clearance value up to $0.07 \mathrm{~mm}$ can be detected using this algorithm. 


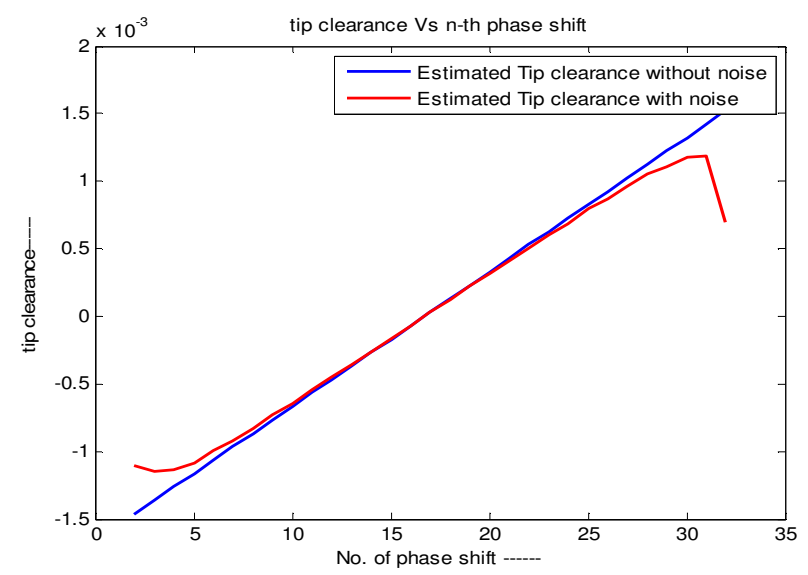

Figure 6. Plot of estimated tip clearance with and without noise

\section{CONCLUSIONS}

In this paper blade tip clearance measurement is found considering the effect of noise resulting from the harsh working environment of the turbine engine.The optimum noise level is found to be $0.05 *$ random noise.

Inclusion of the effects of temperature, stress, vibration effects, and other damaging effects like foreign objects can extend this project further. Clearance measurement data can be analysed along with the life of the turbine blade in gas turbine engine. The noise range can be improved further by employing suitable techniques.

\section{REFERENCES}

[1] MaddalenaVioletti and Anja K. Skrivervik, Qin Xu and Micha"elHafner, "New Microwave Sensing System for Blade Tip Clearance Measurement in Gas Turbines", IEEE SENSORS-2012, Proceedings Pp1397

[2] David Kwapisz, MichaëlHafner, Ravi Rajamani, “Application of Microwave Sensing to Blade Health Monitoring”,European Conference of the Prognostics and Health Management Society, 2012

[3] M. Violetti, J. -F. Z"urcher, J. Geisheimer, and A. K. Skrivervik, "Design of antenna based sensors for blade tip clearance measurement in gas turbines,"Proc. 4th European Conference on Antennas and Propagation-2010, Barcelona, Spain.

[4] A. B. Vakhtin, S.-J. Chen, and S. M. Massick, "Optical probe for monitoring blade tip clearance," 47th AIAA Aerospace Sciences MeetingIncluding The New Horizons Forum and Aerospace Exposition-2009,, Orlando, FL.

[5] TiborFabian, SangkyunKang, FritzPrim ,"Capacitive blade tip clearance measurements for a micro gas turbine",IEEE Instrumentation and Measurement Technology Conference Anchorage-2002, USA.

[6] CatalinMandache, Tyrell Mcelhinney, NezihMrad,"Aircraft Engine Blade Tip Monitoring Using Pulsed Eddy Current Technology”, 4th International Symposium,NDT in Aerospace 2012

[7] Meyer, Richard T.; DeCarlo, Raymond A.; Pekarek, Steve; and Doktorcik, Chris, "Gas TurbineEngine BehavioralModeling" (2014).ECE Technical Reports.Paper 454. 
International Journal of Recent advances in Mechanical Engineering (IJMECH) Vol.4, No.2, May 2015

\section{AUTHORS}

Ms.AnweshaDutta(b. 1991) received B.EElectronics and Telecommunication Engineering from Mumbai University, Mumbai, India in 2012. Currently doing final year M.Tech Communication Engineering in VIT University, Vellore TamilNadu, India. Her area of interest includes radar signal processing and Gas Turbine Engine sensor's signal processing.

Ms.Shivangi (b. 1990) received B.Tech Electronics and Communication Engineering from BijuPatnaik University of Technology, Bhubaneshwar, Orissa, India in 2012. Currently doing final year M.Tech Communication Engineering in VIT University, Vellore TamilNadu, India. Her area of interest includes radar signal processing and Gas Turbine Engine sensor's signal processing.

Dr. J. Valarmathi (b.1968) received B.Tech Electronics from MIT, Anna University in 1992 and completed her M.Tech and PhD from VIT University in 2004 and 2013. Currently working as a professor in VIT University, has 20 years of teaching experience with 50 publications in Journals and conferences. Her research interest includes multi sensor data fusion in radar signals and Gas turbine engines.
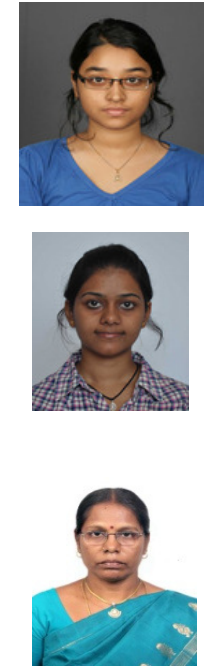